\title{
REG-I $\alpha$ IN THE DIAGNOSIS OF ACUTE CORONARY SYNDROME - A PILOT STUDY
}

\author{
David Stejskal $^{\text {a*}}$, Borek Lacnak ${ }^{\mathrm{a}}$, Michal Karpisek ${ }^{\mathrm{b}}$
}

\author{
a Department of Laboratory Medicine and Internal Department Sternberk Hospital, Czech Republic \\ ${ }^{b}$ Department of Pharmacology, Faculty Veterinary Brno \\ e-mail:david.stejskal@quick.cz
}

Received: April 28, 2006; Accepted: May 20, 2006

Key words: Reg-Ia/Cardiac Troponin/Acute Coronary Syndrome

The commonly used laboratory markers of coronary involvement in subjects with acute coronary syndrome (ACS) are not yet myocardial ischemia-specific and show a late irreversible involvement of the myocardium. A laboratory test has been searched for in order to distinguish persons with myocardial ischemia and typical CAD symptoms to CAD-free individuals. Reg-I $\alpha$ is the product of Reg-I gene which plays a significant role in myocardial regeneration. 38 individuals with suspicion of acute coronary syndrome were tested on admission, after 2 and 6 hours. In all of them cardiac troponin I, myoglobin, C-reactive protein (CRP) and Reg-I $\alpha$ were analysed. Our findings did not support the hypothesis that measurement of Reg-Ia maybe the useful marker of myocardial stress.

\section{INTRODUCTION}

In the last decade, several communications have reported on the need to search for new, sensitive and particularly early tests of myocardial ischemia allowing early and effective therapy. This need arises from the disadvantages of the commonly used laboratory markers of myocardial ischemia (troponins or CK-MB mass):

1. detection of progressed myocardial necrosis

2. insufficient diagnostic value of the assessment of myocardial ischemia

3. elevated values several hours after the onset of myocardial damage.

Of new biomarkers, highly interesting is Reg-I $\alpha$. Recently, the regenerating gene (Reg) has been reported to play an important role in various regenerating tissues, but it is unknown whether the Reg gene can be activated in the heart. Reg gene is located on the chromosome 6, it's official symbol is Reg1 and name is Regenerating islet-derived 1 (synonyms RGPI, RATLITHOST, Reg, LITHOST, RATRGPI, Rgp1;rat regenerating islet-derived mouse homolog 1 , regeneration protein lithostatin pancreatic stone protein). To date, no clinical studies of Reg-I $\alpha$ values in individuals with myocardial damages have been carried out but recent paper demonstrated the $\mathrm{Reg} / \mathrm{Reg}$ receptor system in damaged hearts ${ }^{1}$. Reg-I $\alpha$ could to have a high diagnostic validity for the assessment of myocardial stress. The aim of the present paper was to assess the possible diagnostic utility for the above-mentioned marker in the diagnosis of acute coronary syndromes.

\section{METHODS}

38 patients admitted to the Deptartment of Internal Medicine of Sternberk Hospital with suspect acute coronary syndrome.

Plasma cardiac troponin I (LEIA, DPC), myoglobin (LAT, Denka Seiken), C-reactive protein (CRP) (LAT, Biovendor) and Reg -I $\alpha$ (ELISA, Biovendor) were analysed on admission, after 2 and 6 hours. Plasma creatinine values were analysed on admission (Jaffe, Biovendor).

Diagnosis of ACS was made according to ESC/ACC guidelines.

Table 1. Basic laboratory characteristics of patients.

\begin{tabular}{|l|c|c|c|r|c|}
\hline Parameter & Units & X & Median & SD & Normality \\
\hline Age & years & 72.4 & 73.0 & 8.7 & Yes \\
\hline cTnI & $\mu \mathrm{g} / 1$ & 0.34 & 0.2 & 0.43 & No \\
\hline Myoglobin & $\mathrm{mg} / 1$ & 110.8 & 89.5 & 74.1 & No \\
\hline Reg-I & $\mu \mathrm{g} / 1$ & 910.8 & 697.1 & 729.8 & No \\
\hline Creatinine & $\mu \mathrm{mol} / 1$ & 111.0 & 102.0 & 30.6 & Yes \\
\hline
\end{tabular}

X-mean, SD - standard deviation Normality tested with Komolgorov-Smirnov. 


\section{RESULTS}

Reg-I values had an abnormal distribution (Tab. 1)

Patients with acute coronary syndrome (ACS, $\mathrm{n}=7$, all of had non-STEMI) did not significantly differ from ACS-free individuals $(n=28)$ (Tab. 2).

Table 2. Reg-I $\alpha$ values divided by ACS presence.

\begin{tabular}{|c|c|c|c|c|c|}
\hline Parameter & Group & Median & Normality & $p$ & Normality \\
\hline Reg-I & ACS & 773.2 & No & & No \\
\hline Reg-I & non-ACS & 679.7 & No & 0.48 & No \\
\hline
\end{tabular}

$\mathrm{r}$ - correlation coefficient, $\mathrm{p}$ - probable value

Reg-1 $\alpha$ values correlated significantly with myoglobin $(\mathrm{r}=0.49 ; \mathrm{p}<0.01)$ and CRP $(\mathrm{r}=0.62 ; \mathrm{p}<0.01)$ values. Correlation of Reg- $1 \alpha$ with CRP persisted even after the CRP to myoglobin adjustment $(r=0.57 ; p<0.01)$.

Table 3. Significant relation between Reg-I $\alpha$ and the other analysed parameters.

\begin{tabular}{|c|l|c|c|}
\hline Parameter 1 & Parameter 2 & $\mathrm{r}$ & $\mathrm{p}$ \\
\hline \multirow{2}{*}{ Reg-I } & CRP & 0.62 & 0.0005 \\
\cline { 2 - 4 } & Myoglobin & 0.49 & 0.0002 \\
\cline { 2 - 4 } & CRP adj with myoglobin & 0.59 & 0.0013 \\
\hline
\end{tabular}

Adjustment ((CRP - 1.88) / myoglobin. From regression aquation. F ratio $9.3, \mathrm{p}=0.005)$

$\mathrm{r}$ - correlation coefficient, $\mathrm{p}$ - probable value

In stepwise regression age and myoglobin were not included in the calculation. The regression equation was: Reg-I $=8.7$ * creatinine $+19.4 *$ CRP $-965.5 *$ cTnI 354.5 (F ratio 22.1, p $<0.01$, coefficient of determination $0.69)$.

\section{DISCUSSION}

In 1984, a novel gene was found, which plays a role in various types of tissue regeneration.

Recently, the regenerating gene (Reg) has been documented to play an important role in the regeneration of myocardium and transcriptional activation of Reg was detected in the heart in response to heart stress ${ }^{1-3}$. In damaged human myocardium (myocardial infarction) Reg-1 gene expression in fine granular pattern in the cytoplasm of cardiomyocytes was located. Reg protein secretion into the serum after ischemia, and circulating levels of the protein after myocardial infarction were higher than those after aortic constriction. These results demonstrated the presence of the $\mathrm{Reg} / \mathrm{Reg}$ receptor system in damaged hearts. In view of emerging evidence of Reg for tissue regeneration in a variety of tissues/organs, it is proposed that the damaged heart may be a target for Reg action and that Reg may protect against acute heart stress ${ }^{3}$.

Our results failed verify to the recent hypothesis about the use of serum Reg-I $\alpha$ analyses in diagnosis of myocardial stress (acute coronary syndrome).

In conclusion, the pilot study indicates that Reg-I $\alpha$ analysis is not useful in early differential diagnosis of acute coronary syndrome. Reg-I $\alpha$ strongly correlated with CRP. It could be postulated that Reg-I $\alpha$ values are regulated via systemic inflammation.

\section{REFERENCES}

1. Fukui H, Fujii S, Takeda J, Kayahara T, Sekikawa A, Nanakin A, Suzuki K, Hisatsune H, Seno H, Sawada M, Fujimori T, Chiba T (2004) Expression of reg I alpha protein in human gastric cancers. Digestion 69, 177-184.

2. Sanchez D, Gmyr V, Kerr-Conte J, Kloppel G, Zenilman ME, GuyCrotte O, Pattou F, Figarella C (2004) Implication of Reg I in human pancreatic duct-like cells in vivo in the pathological pancreas and in vitro during exocrine dedifferentiation. Pancreas 29,14-21.

3. Kiji T, Dohi Y, Takasawa S, Okamoto H, Nonomura A, Taniguchi $S$ (2005) Activation of regenerating gene Reg in rat and human hearts in response to acute stress. Am J Physiol Heart Circ Physiol., 289, 277-284. 